\title{
Extension of the Module of Invertible Transformations. Classification of Integrable Systems
}

\author{
A. V. Mikhailov ${ }^{1}$, A. B. Shabat ${ }^{2}$, and R. I. Yamilov ${ }^{2}$ \\ 1 L. D. Landau Institute for Theoretical Physics, Academy of Sciences of USSR, Kosygina 2, Moscow, \\ V334, USSR \\ 2 Department of Physics and Mathematics, Bashkirian Branch of the Academy of Sciences of the \\ USSR, Tukaeva 50, UFA, USSR
}

\begin{abstract}
We demonstrate that for the systems of equations, which are invariant under a point group or possess conservation laws of the zeroth or first order, a nontrivial extension of the module of invertible transformations is possible. That simplifies greatly a classification of the integrable systems of equations. Here we present an exhaustive list and a classification of the second order systems of the form $u_{t}=u_{x x}+f\left(u, v, u_{x}, v_{x}\right),-v_{t}=v_{x x}+g\left(u, v, u_{x}, v_{x}\right)$, which possess the conservation laws of higher order. The reduction group approach allows us to define the Lax type representations for some new equations of our list.
\end{abstract}

\section{Introduction}

The systems of evolution equations, related by the invertible transformations, should be considered as equivalent ones. In many applications there occurs a situation when a system of equations possess a continuous point symmetry group, and it is sufficient to restrict ourselves to a reduced subset of dynamical variables, consisting of the group invariants. With the accuracy up to the unessential constants of integration, which can be removed by the transformations of the group, a reduced subset contains all the information about a general solution. Thus, we can also consider two systems of equations as equivalent ones, if their reduced subsets of dynamical variables are related by invertible substitutions. In contrast to the point transformations, such substitutions may violate local conservations laws. We shall study the most interesting substitutions, that preserve the locality property of the conservation laws (recall, the conservation law $\rho_{t}=D \sigma$ is called local, if $\rho$ and $\sigma$ are the functions of a finite number of the dynamical variables). The considered module extension of the invertible substitutions simplifies drastically the classification of the integrable equations. The use of these substitutions allowed us to make a list of integrable systems of equations $[1,2,3]$ more comprehensible. Many of the well-known equations have proved to be equivalent by this extended 
module. We also give a list of substitutions which connect the equations of the list. In the end of Sect. II several transformations of the Miura type are also given. They make it possible to expose additional connections between the equations which are not related between each other by invertible substitutions. The last section is devoted to the description of the $\mathrm{L}-\mathrm{A}$ pairs which are necessary in order to use the inverse scattering transforms. As it is known, the presence of the commutational representation is a sufficient integrability condition of the system of equations.

\section{Extension of the Module of the Invertible Transformations}

1. For definiteness, in this paper we shall consider the system of equations of the form:

$$
u_{t}=u_{x x}+f\left(u, v, u_{x}, v_{x}\right), \quad-v_{t}=v_{x x}+g\left(u, v, u_{x}, v_{x}\right) .
$$

In particular, the systems of the two equations of the form

$$
\mathbf{u}_{t}=A(\mathbf{u}) \mathbf{u}_{x x}+\mathbf{F}\left(\mathbf{u}, \mathbf{u}_{x}\right)
$$

possessing local conservation laws of sufficiently high order can be reduced to the form (1.1) (See [1]).

The invertible substitutions of variables, that do not change the form of system (1.1), are exhausted by the composition of the elementary substitutions $x \rightarrow a x+b t+c, t \rightarrow a^{2} t+d(a, b, c, d \in \mathbb{C})$ conformal transformations $u \rightarrow U(u)$, $v \rightarrow V(v)$ and involution

$$
u \rightarrow v, \quad v \rightarrow u, \quad t \rightarrow-t, \quad x \rightarrow-x .
$$

The system of evolution equations is extended up to an infinite dynamical system in variables $u, v, u_{1}, v_{1}, u_{2}, v_{2}, \ldots$, where $u_{1}=u_{x}, v_{1}=v_{x}, u_{2}=u_{x x}$, and so on, which form a complete set of dynamical variables. Systems (1.1), that are invariant under the continuous conformal group action can be transformed using conformal substitutions and involution (1.2) to a special form, such as

$$
f=f\left(\varepsilon u+v, u_{1}, v_{1}\right), \quad g=g\left(\varepsilon u+v, u_{1}, v_{1}\right), \quad \varepsilon=0,1 .
$$

For instance, the famous nonlinear Schrödinger equation $i \psi_{t}=\psi_{x x}+|\psi|^{2} \psi$ is sure to be reduced to the form (1.3) with $\varepsilon=1$ :

$$
u_{t}=u_{2}+u_{1}^{2}+\exp (u+v), \quad-v_{t}=v_{2}+v_{1}^{2}+\exp (u+v) .
$$

If the group of point symmetries is nonabelian, then the reduction to this form is not unique. The Heisenberg model can serve as an example, of the system that can be reduced to the form (1.3) in two different ways (see below).

Further the maximal subset of dynamic variables $\varepsilon u+v, u_{1}, v_{1}, \ldots$ that are invariant under the one-parametric point group transformations we shall call as $a$ reduced subset of dynamical variables. Both reduced and complete subsets are generated by the action of the operator $D=u_{1} \partial / \partial u+v_{1} \partial / \partial v+u_{2} \partial / \partial u_{1}+\cdots$ on the generators. For the generators of a complete set of one should choose $u, v$, and for a reduced subset we shall choose variables $\varepsilon u+v, u_{1}$. The considered substitutions 
will have the form $\varepsilon u+v=Z(y, z) \quad u_{1}=W(y, z)$, where $Z, W$, are functionally independent functions of the generators of a reduced $y=\varepsilon u+v, z=u_{1}$ (or a complete, i.e. $y=u, z=v$ ) subset of dynamical variables. We shall require that the function $W$ should be a density of the conservation law of the initial system (1.3) (or (1.1)). That will allow us to redetermine the evolution in time of a complete set of dynamical variables.

The example of the invertible substitution defined on the reduced subsets is $U_{1}=\exp (u+v), U+V=2 u_{1}$, which relates the nonlinear Schrödinger equation (1.4) to the system $U_{t}=U_{2}+(U+V) U_{1},-V_{t}=V_{2}-(U+V) V_{1}$, the last one is equivalent to the famous Kaup equation. (Below we will denote such transformations as $u_{1} \rightarrow \exp (u+v), u+v \rightarrow 2 u_{1}$.) Let's point out that this substitution is not the transformation of the Miura type, because the latter is not invertible within the finite subsets of dynamical variables.

There is an important class of invertible substitution that relates reduced and complete subsets of dynamical variables:

$$
u \rightarrow Z\left(\varepsilon u+v, u_{1}\right) \quad v \rightarrow W(\varepsilon u+v),
$$

where $Z, W$, are any functions $\left(d Z / d v_{1} d W / d v \neq 0\right)$. In general, substitution (1.5) spoils the form (1.1) of the original system. This form is to be preserved iff

$$
\partial^{2} f / \partial v_{1}{ }^{2}=0, \quad 2 \partial Z / \partial v=\partial f / \partial v_{1} \partial Z / \partial u_{1} .
$$

Consider the system of equations $u_{t}=u_{2}+2 v v_{1},-v_{t}=v_{2}-u_{1}$, that relate to the system $u_{t}=u_{2}+v_{1}{ }^{2}+v\left(u-v^{2} / 2\right),-v_{t}=v_{2}-u+v^{2} / 2$ by the substitution $Z=u_{1}+v^{2} / 2, W=v$. The initial system of equations which can be reduced to the Bousinesque equation after excluding the variable $u$, possesses local conservation laws of an arbitrary high order. Using the results of paper [1] one can check easily that the obtained system does not possess local conservation laws. Below we shall obtain sufficient conditions so that the local conservation laws would not be violated.

Let us consider the following change of variables:

$$
u_{1} \rightarrow \rho(u, v), \quad \varepsilon u+v \rightarrow \chi(v),
$$

where $\rho$ is a density of the conservation law. This substitution is admissible for the class of system of the form (1.1) iff

$$
\partial^{2} g / \partial u_{1}{ }^{2}=\partial^{2} g / \partial u_{1} \partial v_{1}=\partial\left[\left(\partial g / \partial u_{1}\right) /(\partial \rho / \partial u)\right] / \partial u=0
$$

and

$$
\begin{array}{ll}
\varepsilon=0, \chi=v, & \text { if } \partial g / \partial u_{1}=0 \\
\varepsilon=1, \partial \chi / \partial v=-\left(\partial g / \partial u_{1}\right) /(\partial \rho / \partial u) & \text { if } \partial g / \partial u_{1} \neq 0 .
\end{array}
$$

Let's note that substitutions (1.7) are inverse to the substitution of the from (1.5). It is evident that substitutions of the form (1.7) preserve the locality property of the conservations laws and increase their order by one.

2. Here we shall consider the systems of equations of the form:

$$
u_{t}=u_{2}+f\left(u+v, u_{1}, v_{1}\right), \quad-v_{t}=v_{2}+g\left(u+v, u_{1}, v_{1}\right) \text {. }
$$


They are invariant under involution (1.2). We shall call them symmetrical systems. For symmetrical systems we shall take the substitutions which do preserve the property of the higher order conservation laws to be local. Below we shall refer to the lemma about the invariant functions. A function $g$ we call invariant if it is not changed under involution (1.2) and $\partial g / \partial u=\partial g / \partial v$.

Lemma. If the local conservation law $\rho_{t}=D \sigma$ of the system (1.8) possesses invariant density $\rho$, then function $\sigma$ is also invariant.

Let's consider the following change of the reduced subsets of the dynamical variables:

$$
u+v \rightarrow p(u+v), \quad u_{1} \rightarrow p^{\prime}(u+v) u_{1}+q(u+v), \quad p^{\prime} \neq 0 .
$$

We require that the function $p^{\prime} u_{1}+q$ should be a density, therefore $\left[p^{\prime}\left(u_{1}-\right.\right.$ $\left.\left.v_{1}\right)+2 q\right]_{t}=D \sigma$, and $\sigma$ is an invariant function as it follows from the lemma. The evolution of a complete set of variables is defined from the relationships $u_{t}+v_{t}=p_{t}$, $u_{t}-v_{t}=\sigma$. It is easy to check that the obtained system is also symmetrical.

Theorem 1. Substitution (1.9) defines the equivalence relation on the set of symmetrical systems.

Therefore, the systems that relate to substitution (1.9) we shall call symmetrically equivalent. To construct the equivalence class of a symmetrical system one should find all the densities of the form $p^{\prime}(u+v) u_{1}+q(u+v)$. If there are no nontrivial densities, then the equivalent class can be determined by the substitution $u_{1} \rightarrow u_{1}+\mu, v_{1} \rightarrow v_{1}-\mu$.

Let us consider two examples. The system of the form (1.1) with

$$
f=2 a u v u_{1}+b u^{2} v_{1}+b(a-b) u^{3} v^{2} / 2+c u^{2} v, \quad g=f^{*}
$$

(where $f^{*}$ denotes the result of involution (1.2)) by the conformal substitution $u \rightarrow \log u, v \rightarrow \log v$ is reduced to the form (1.8), where

$$
f=u_{1}{ }^{2}+\left(2 a u_{1}+b v_{1}+c\right) \exp (u+v)+\frac{1}{2} b(a-b) \exp 2(u+v) .
$$

Using the conservation law of the system (1.11) with the density $\alpha u_{1}+\beta \exp (u+v)$, $\alpha, \beta \in \mathbb{C}$, it is not difficult to check that if $b=2 a, c=0$ this system is symmetrically equivalent to a linear one, if $b=2 a, c \neq 0$ it is equivalent to the nonlinear Schrödinger equation (1.3), and if $b \neq 2 a$ it is equivalent to the derivative nonlinear Schrödinger equation (i.e. to system (1.8), (1.11) $a=b=1, c=0$ ). Some particular cases of the system (1.1), (1.10) were studied by many authors from the viewpoint of the inverse scattering transform. In these cases a special gauge transformation results in substitutions of the form (1.9). In our approach the substitutions are defined by the classical symmetries and conservation laws. As a second example, let's establish the relationship between the known systems:

$$
\begin{aligned}
& u_{t}=u_{2}-2 u_{1}{ }^{2} /(u+v), \quad-v_{t}=v_{2}-2 v_{1}{ }^{2} /(u+v) ; \\
& u_{t}=u_{2}-u_{1}{ }^{2}+2 u_{1} v_{1}, \quad-v_{t}=v_{2}-v_{1}{ }^{2}+2 v_{1} u_{1} .
\end{aligned}
$$

The system of equations (1.12) is obtained from the well known integrable 
Heisenberg model $\mathbf{S}_{t}=\mathbf{S} \times \mathbf{S}_{x x},{S_{1}}^{2}+{S_{2}}^{2}+{S_{3}}^{2}=1$ by the point transformation:

$$
S_{1}=2 /(u+v), \quad S_{2}=2 u v /(u+v), \quad S_{3}=(u-v) /(u+v) .
$$

System (1.13) has been studied by many authors via the inverse scattering transform. The substitution of the form (1.9) $u+v \rightarrow \log (u+v), u_{1} \rightarrow u_{1} /(u+v)$ transforms the system of equations (1.12) into (1.13).

These examples show that the systems not related to each other at first glance, might prove symmetrically equivalent. In the case of the system (1.8) with $\partial^{2} f / \partial v_{1}^{2}=0$ the following theorem yields a simple criterion of equivalence.

Theorem 2. Two systems of equations of the form (1.8) with $\partial^{2} f / \partial v_{1}{ }^{2}=0$ are symmetrically equivalent if and only if substitution (1.5), (1.6) with $\varepsilon=1$ makes them conformally equivalent.

The theorem means that substitution (1.9) is nothing but a composition of two substitutions of the form (1.5), (1.6) with $\varepsilon=1$ and the conformal transformation.

It follows from the lemma that substitution (1.9) allows one to recount the local conservation laws if the densities are invariant. The following theorem shows that really conservation laws of higher order can be considered as invariant ones.

Theorem 3. Let system (1.8) have at least two local conservation laws of higher order and $\rho$ be the density of the conservation law of the order $N \geqq 2$. Then the density $\rho+\rho^{*}$ is of the order $N$ and invariant modulo of total derivatives and densities of the order not higher than one.

Corollary. If a symmetrical system possesses local conservation laws of higher order, then the system of equations, obtained as a result of the substitution of the form (1.9) or (1.5), (1.6) with $\varepsilon=1$, also possesses this property.

For example, the substitution of variables $u \rightarrow 2 i u, v \rightarrow i \tanh (u+v)$ in the following system:

$$
u_{t}=u_{2}-2 \tanh (u+v) u_{1}^{2}, \quad-v_{t}=v_{2}-2 \tanh (u+v) v_{1}^{2},
$$

results in the system

$$
u_{t}=u_{2}+D\left(u^{2} v\right), \quad-v_{t}=v_{2}-D\left(v^{2} u+\mu u\right)
$$

where $\mu=1$. The system (1.15) relates to (1.12) by the conformal transformation, hence on account of the consequence of Theorem 3 system (1.16) possesses an infinite set of local conservation laws. One can make the substitution $u \rightarrow u_{1}$, $v \rightarrow-2 /(u+v)$ directly in system (1.12), in this case one obtains system (1.16) with $\mu=0$, i.e. the derivative nonlinear Schrödinger equation. Systems (1.16) with $\mu=0$ and $\mu=1$ are not point or symmetrically equivalent.

\section{Classification of Integrable Systems of the Form (1.1)}

1. In our previous papers $[1,2,3]$ there have been obtained the necessary conditions that the systems of equations of the form (1.1) possess the local conservation laws of higher order. These conditions proved to be so effective that they allowed us to determine completely a possible form of the right-hand sides of the systems. 
Among them quite a lot of systems proved to be symmetrical. The afore-described module extension allows one to reduce drastically a list of integrable equations: it is just enough to give a representative from each symmetrical class. Below we shall present an exhaustive list of integrable systems of equations of the form (1.1).

List of integrable systems of equations.

(a)

$$
\begin{aligned}
u_{t} & =u_{2}+u_{1}^{2}+v_{1}, \\
-v_{t} & =v_{2}-2 u_{1} v_{1} ;
\end{aligned}
$$

(A)

$$
\begin{aligned}
u_{t} & =u_{2}+D\left(u^{2}+v\right), \\
-v_{t} & =v_{2}-2 D(u v) ;
\end{aligned}
$$

(b) $\quad u_{t}=u_{2}+u^{2} v$

$$
-v_{t}=v_{2}+v^{2} u
$$

(c) $\quad u_{t}=u_{2}+(u+v) u_{1}$,

$$
-v_{t}=v_{2}-(u+v) v_{1} \text {; }
$$

(d) $\quad u_{t}=u_{2}+u_{1}{ }^{2} v_{1}-4 v_{1}$,

$$
-v_{t}=v_{2}-v_{1}^{2} u_{1}+4 u_{1} \text {; }
$$

(D) $\quad u_{t}=u_{2}+D\left(u^{2} v-4 v\right)$,

$$
-v_{t}=v_{2}-D\left(v^{2} u-4 u\right) \text {; }
$$

(d') $\quad u_{t}=u_{2}-(u+v)^{-2} u_{1}{ }^{2} v_{1}-2(u+v)^{-1} u_{1}{ }^{2}$,

$$
-v_{t}=v_{2}+(u+v)^{-2} v_{1}{ }^{2} u_{1}-2(u+v)^{-1} v_{1}^{2} \text {; }
$$

(d") $\quad u_{t}=u_{2}+\operatorname{sech}^{2}(u+v) u_{1}{ }^{2} v_{1}-2 \tanh (u+v) u_{1}{ }^{2}$, $-v_{t}=v_{2}-\operatorname{sech}^{2}(u+v) v_{1}^{2} u_{1}-2 \tanh (u+v) v_{1}^{2}$;

(e) $\quad u_{t}=u_{2}-2 \tanh (u+v)\left(u_{1}^{2}-4\right)$, $-v_{t}=v_{2}-2 \tanh (u+v)\left(v_{1}^{2}-4\right)$;

(f) $\quad u_{t}=u_{2}-2(u+v)^{-1} u_{1}^{2}-4(u+v)^{-2}\left[2(1+u v) u_{1}+\left(1-u^{2}\right) v_{1}\right]$, $-v_{t}=v_{2}-2(u+v)^{-1} v_{1}^{2}+4(u+v)^{-2}\left[2(1+u v) v_{1}+\left(1-v^{2}\right) u_{1}\right]$

(g) $\quad u_{t}=u_{2}+u_{1}^{2} v_{1}$, $-v_{t}=v_{2}-v_{1}^{2} u_{1}-u_{1}$

(G) $u_{t}=u_{2}+D\left(u^{2} v\right)$,

$$
-v_{t}=v_{2}-D\left(v^{2} u+u\right) \text {; }
$$

(h) $u_{t}=u_{2}+u_{1}^{2}-2 u_{1} v_{1}$,

$$
-v_{t}=v_{2}-v_{1}^{2}-2 v_{1} u_{1} \text {; }
$$

(H) $u_{t}=u_{2}+D\left(u^{2}-2 u v\right)$, $-v_{t}=v_{2}-D\left(v^{2}-2 u v\right)$; 
(h') $\quad u_{t}=u_{2}-2(u+v)^{-1} u_{1}^{2}$,

$$
-v_{t}=v_{2}-2(u+v)^{-1} v_{1}^{2}
$$

(h") $\quad u_{t}=u_{2}-2 \tanh (u+v) u_{1}{ }^{2}$,

$$
-v_{t}=v_{2}-2 \tanh (u+v) v_{1}^{2}
$$

(i) $\quad u_{t}=u_{2}+u_{1}{ }^{2} v_{1}$,

$$
-v_{t}=v_{2}-v_{1}^{2} u_{1}
$$

(I) $\quad u_{t}=u_{2}+D\left(u^{2} v\right)$,

$$
-v_{t}=v_{2}-D\left(v^{2} u\right)
$$

(j) $\quad u_{t}=u_{2}-2(u+v)^{-1}\left(u_{1}^{2}+1\right)$,

$$
\begin{aligned}
u_{t} & =u_{2}+\exp (u+v) u_{1}{ }^{2} v_{1}+u_{1}{ }^{2}, \\
-v_{t} & =v_{2}-\exp (u+v) v_{1}{ }^{2} u_{1}+v_{1}{ }^{2} ;
\end{aligned}
$$

$$
-v_{t}=v_{2}-2(u+v)^{-1}\left(v_{1}^{2}+1\right)
$$

(k) $\quad u_{t}=u_{2}-2(u+v)^{-1} u_{1}^{2}-4(u+v)^{-2}\left[(u-v) u_{1}+u v_{1}\right]$,

$$
-v_{t}=v_{2}-2(u+v)^{-1} v_{1}^{2}+4(u+v)^{-2}\left[(u-v) v_{1}-v u_{1}\right] \text {; }
$$

(l) $\quad u_{t}=u_{2}+R(y) u_{1}{ }^{2} v_{1}+R^{\prime}(y) u_{1}{ }^{2}-2 / 3\left[R^{\prime \prime}(y)-2 c\right] u_{1}+1 / 3 R^{\prime \prime \prime}(y)$,

$$
-v_{t}=v_{2}-R(y) v_{1}^{2} u_{1}+R^{\prime}(y) v_{1}^{2}+2 / 3\left[R^{\prime \prime}(y)-2 c\right] v_{1}+1 / 3 R^{\prime \prime \prime}(y),
$$

where $\left.y=y(u+v), y^{\prime}=R(y) \neq 0, R(y)=a y^{4}+b y^{3}+c y^{2}+d y+e\right)$;

(m)

$$
\begin{aligned}
u_{t} & =u_{2}-2(u+v)^{-1} u_{1}{ }^{2}-4\left[P(u, v) u_{1}+R(u) v_{1}\right](u+v)^{-2}, \\
-v_{t} & =v_{2}-2(u+v)^{-1} v_{1}{ }^{2}+4\left[P(u, v) v_{1}+R(-v) u_{1}\right](u+v)^{-2}
\end{aligned}
$$

(n)

$$
\begin{aligned}
u_{t} & =u_{2}-2(u+v)^{-1}\left[u_{1}{ }^{2}+R(u)\right]+R^{\prime}(u) / 2, \\
-v_{t} & =v_{2}-2(u+v)^{-1}\left[v_{1}{ }^{2}+R(-v)\right]-R^{\prime}(-v) / 2 ;
\end{aligned}
$$

(in Eqs. (m), (n): $P(u, v)=2 a u^{2} v^{2}+b\left(u v^{2}-v u^{2}\right)-2 c u v+d(u-v)+2 e, R(z)=a z^{4}+$ $\left.b z^{3}+c z^{2}+d z+e\right)$

$$
\begin{aligned}
u_{t} & =u_{2}+\exp (\phi)\left(u_{1}^{2}+1\right) v_{1}+\phi_{v} u_{1}^{2}+2 r u, \\
-v_{t} & =v_{2}-\exp (\phi)\left(v_{1}{ }^{2}+1\right) u_{1}+\phi_{v} v_{1}^{2}-2 r v_{1} ;
\end{aligned}
$$

(p)

$$
\begin{aligned}
u_{t} & =u_{2}+\exp (\phi)\left(u_{1}{ }^{2}+1\right) v_{1}+\phi_{u}\left(u_{1}^{2}+1\right), \\
-v_{t} & =v_{2}-\exp (\phi)\left(v_{1}{ }^{2}+1\right) u_{1}+\phi_{v}\left(v_{1}{ }^{2}+1\right),
\end{aligned}
$$

(in Eqs. (o), (p): $\exp (\phi)=y(u+v)-y(u-v), r=y(v+v)+y(u-v), y^{\prime} \neq 0,\left(y^{\prime}\right)^{2}=$ $-4 y^{4}+a y^{3}+b y^{2}+c y+d$, or $\left(y^{\prime}\right)^{2}=-y^{4}+a y^{3}+b y^{2}+c y+d$ respectively);

(q)

$$
\begin{aligned}
u_{t} & =u_{2}+v_{1}, \\
-v_{t} & =v_{2}-u_{1}^{2} ;
\end{aligned}
$$

(Q)

$$
\begin{aligned}
u_{t} & =u_{2}+v_{1}, \\
-v & =v_{2}-D\left(u^{2}\right) ;
\end{aligned}
$$


(r)

$$
\begin{aligned}
u_{t} & =u_{2}+(u+v)^{2}, \\
-v_{t} & =v_{2}+(u+v)^{2} ; \\
u_{t} & =u_{2}+(u+v) v_{1}-(u+v)^{3} / 6 \\
-v_{t} & =v_{2}-(u+v) u_{1}-(u+v)^{3} / 6 ; \\
u_{t} & =u_{2}+v_{1} \\
-v_{t} & =v_{2}-u_{1}{ }^{2}-\left(v+u^{2} / 2\right) u_{1} ;
\end{aligned}
$$

(s)

there are six equations of the form:

(u)

$$
\begin{aligned}
u_{t} & =u_{2}+v_{1}^{2}+\theta_{u} v_{1}+z_{v}, \\
-v_{t} & =v_{2}+u_{1}^{2}-\theta_{v} u_{1}+z_{u} ;
\end{aligned}
$$

where

$$
\begin{aligned}
\theta= & a \exp [-(u+v)]+a_{1} \exp \left(-\omega u-\omega^{*} v\right)+a_{2} \exp \left(-\omega^{*} u-\omega v\right), \\
z= & b \exp (u+v)+b_{1} \exp \left(\omega u+\omega^{*} v\right)+b_{2} \exp \left(\omega^{*} u+\omega v\right) \\
& +c \exp [-2(u+v)]+c_{1} \exp \left[-2\left(\omega u+\omega^{*} v\right)\right] \\
& +c_{2} \exp \left[-2\left(\omega^{*} u+\omega v\right)\right]
\end{aligned}
$$

and $\omega=\exp (2 \pi i / 3), \omega^{*}=\exp (-2 \pi i / 3)$ :

(u1)

$$
\begin{aligned}
u_{t} & =u_{2}+v_{1}^{2}, \\
-v_{t} & =v_{2}+u_{1}^{2} ; \\
u_{t} & =u_{2}+2 v v_{1}, \\
-v_{t} & =v_{2}+2 u u_{1} ;
\end{aligned}
$$

$$
\begin{aligned}
u_{t} & =u_{2}+v_{1}^{2}+b \exp (u+v)-2 c \exp [-2(u+v)], \\
-v_{t} & =v_{2}+u_{1}^{2}+b \exp (u+v)-2 c \exp [-2(u+v)] ;
\end{aligned}
$$

$$
\begin{aligned}
u_{t}= & u_{2}+v_{1}^{2}-\left\{a \exp [-(u+v)]+\omega a_{1} \exp \left(-\omega u-\omega^{*} v\right)\right. \\
& \left.+\omega^{*} a_{2} \exp \left(-\omega^{*} u-\omega v\right)\right\} v_{1}, \\
v_{t}= & v_{2}+u_{1}{ }^{2}+\left\{a \exp [-(u+v)]+\omega^{*} a_{1} \exp \left(-\omega u-\omega^{*} v\right)\right. \\
& \left.+\omega a_{2} \exp \left(-\omega^{*} u-\omega v\right)\right\} u_{1} ; \\
u_{t}= & u_{2}+v_{1}^{2}-2 c \exp [-2(u+v)]-2 \omega^{*} c_{1} \exp \left[-2\left(\omega u+\omega^{*} v\right)\right] \\
& -2 \omega c_{2} \exp \left[-2\left(\omega^{*} u+\omega v\right)\right], \\
-v_{t}= & v_{2}+u_{1}{ }^{2}-2 c \exp [-2(u+v)]-2 \omega c_{1} \exp \left[-2\left(\omega u+\omega^{*} v\right)\right] \\
& -2 \omega^{*} c_{2} \exp \left[-2\left(\omega^{*} u+\omega v\right)\right] ;
\end{aligned}
$$$$
u_{t}=u_{2}+v_{1}^{2}+b \exp (u+v)+\omega^{*} b_{1} \exp \left(\omega u+\omega^{*} v\right)+\omega b_{2} \exp \left(\omega^{*} u+\omega v\right)
$$$$
-v_{t}=v_{2}+u_{1}^{2}+b \exp (u+v)+\omega b_{1} \exp \left(\omega u+\omega^{*} v\right)+\omega^{*} b_{2} \exp \left(\omega^{*} u+\omega v\right) ;
$$

$$
\begin{aligned}
u_{t}= & u_{2}+v_{1}{ }^{2}-\left\{a \exp [-(u+v)]+\omega a_{1} \exp \left(-\omega u-\omega^{*} v\right)\right. \\
& \left.+\omega^{*} a_{2} \exp \left(-\omega^{*} u-\omega v\right)\right\} v_{1}-\left\{a_{1} a_{2} \exp (u+v)\right. \\
& +\omega^{*} a a_{2} \exp \left(\omega u+\omega^{*} v\right)+\omega a a, \exp \left(\omega^{*} u+\omega v\right)+a^{2} \exp [-2(u+v)] \\
& \left.+\omega^{*} a_{1}{ }^{2} \exp \left[-2\left(\omega u+\omega^{*} v\right)\right]+\omega a_{2}{ }^{2} \exp \left[-2\left(\omega^{*} u+\omega v\right)\right]\right\} / 6 ;
\end{aligned}
$$




$$
\begin{aligned}
-v_{t}= & v_{2}+u_{1}^{2}+\left\{a \exp [-(u+v)]+\omega^{*} a_{1} \exp \left(-\omega u-\omega^{*} v\right)\right. \\
& \left.+\omega a_{2} \exp \left(-\omega^{*} u-\omega v\right)\right\} u_{1}-\left\{a_{1} a_{2} \exp (u+v)\right. \\
& +\omega a a_{2} \exp \left(\omega u+\omega^{*} v\right)+\omega^{*} a a_{1} \exp \left(\omega^{*} u+\omega v\right)+a^{2} \exp [-2(u+v)] \\
& \left.+\omega a_{1}{ }^{2} \exp \left[-2\left(\omega u+\omega^{*} v\right)\right]+\omega^{*} a_{2}{ }^{2} \exp \left[-2\left(\omega^{*} u+\omega v\right)\right]\right\} / 6 ; \\
u_{t}= & u_{2}-(u+v)^{-1}\left(u_{1}^{2}+2 u_{1} v_{1}\right) / 2+a(u+v), \\
-v_{t}= & v_{2}-(u+v)^{-1}\left(v_{1}^{2}+2 v_{1} u_{1}\right) / 2+b(u+v) ; \\
u_{t}= & u_{2}+D\left(u^{2}+v^{-1}\right), \\
-v_{t}= & v_{2}-2 D(u v)-1 .
\end{aligned}
$$

(w)

The equations, denoted by one and the same letters, but differing in a number of primes, are conformally or symmetrically equivalent. For instance, the systems $\left(\mathrm{h}^{\prime}\right)$ and $\left(\mathrm{h}^{\prime \prime}\right)$ are conformally equivalent, but, as has been already pointed out, they belong to different symmetrical classes, and systems $(h),\left(h^{\prime}\right)$ are symmetrically equivalent, but are not related to each other by the conformal transformation.

2. The substitutions of the form (1.5), (1.6) allow us to establish some additional relationships between the equations.

The list of changes of variables
(a) $\rightarrow$ (b)
$u \rightarrow \exp (u)$
$v \rightarrow \exp (-u) v_{1}$
(b) $\rightarrow(\mathrm{A})$
$u \rightarrow u_{1} / u$,
$v \rightarrow u v$;
(c) $\rightarrow$ (A)
$u \rightarrow(u+v) / 2$,
$v \rightarrow-v_{1}$;
(a) $\rightarrow$ (c) $u \rightarrow 2 u_{1}+v$,
$v \rightarrow-v$;
(d) $\rightarrow(\mathrm{e})$
$u \rightarrow \operatorname{arctanh}\left(u_{1} / 2\right)-v$,
$v \rightarrow v$
$(\mathrm{e}) \rightarrow$ (D) $\quad u \rightarrow 2 \tanh (u+v)$,
$v \rightarrow v_{1}$;
$\left(d^{\prime \prime}\right) \rightarrow(f)$
$u \rightarrow \tanh (u+v)$,
$v \rightarrow-\tanh (u+v)-2 / v_{1}$;
$(\mathrm{g}) \rightarrow(\mathrm{j})$
$u \rightarrow 2 / u_{1}-v$,
$v \rightarrow v$
$(\mathrm{j}) \rightarrow(\mathrm{G})$
$u \rightarrow 2(u+v)^{-1}$,
$(\mathrm{g}) \rightarrow\left(\mathrm{h}^{\prime \prime}\right)$
$u \rightarrow i u / 2$,
$\left(\mathrm{h}^{\prime \prime}\right) \rightarrow(\mathrm{G})$
$u \rightarrow 2 i u_{1}$,
$v \rightarrow v_{1}$
$\left(\mathrm{h}^{\prime}\right) \rightarrow(\mathrm{I})$
$u \rightarrow 2(u+v)^{-1}$,
$v \rightarrow i u / 2+\operatorname{arctanh}\left(-i v_{1}\right)$;
$v \rightarrow i \tanh (u+v)$;
(I) $\rightarrow(\mathrm{H})$
$u \rightarrow-u v / 2$,
$v \rightarrow v_{1}$;
(i) $\rightarrow\left(\mathrm{h}^{\prime}\right)$
$u \rightarrow 2 / u_{1}-v_{1}$
$v \rightarrow-u v / 2-v_{1} / v$;
$\left(\mathrm{i}^{\prime}\right) \rightarrow(\mathrm{k})$
$u \rightarrow \exp (u+v)$,
$v \rightarrow v$
$v \rightarrow-2 / v_{1}-\exp (u+v)$;
$(\mathrm{l}) \rightarrow(\mathrm{m})$
$u \rightarrow y(u+v)$,
$v \rightarrow-2 / v_{1}-y(u+v)$;
$(\mathrm{q}) \rightarrow(\mathrm{r})$
$u \rightarrow\left(2 u_{1}+v\right) / 4$,
$v \rightarrow-v / 4$;
$(\mathrm{r}) \rightarrow(\mathrm{Q})$
$u \rightarrow 2(u+v)$,
$v \rightarrow-4 v_{1}$;
$(\mathrm{s}) \rightarrow(\mathrm{t})$
$u \rightarrow-(u+v)$,
$v \rightarrow 2 v_{1}-(u+v)^{2} / 2$;

the following changes of variables relate $(\mathrm{v})$ and $(\mathrm{w})$ to linear and split systems: 
(lin)

$$
\begin{aligned}
u_{t} & =u_{2}+v_{1}+(a-b) u / 2, \\
-v_{t} & =v_{2}-2 b u_{1}+(a-b) v / 2
\end{aligned}
$$

(spl)

$$
\begin{aligned}
u_{t} & =u_{2}+v^{-1}, \\
-v_{t} & =v_{2},
\end{aligned}
$$

respectively:

$$
\begin{array}{lll}
(\mathrm{v}) \rightarrow(\text { lin }) & u \rightarrow 2(u+v)^{1 / 2} & v \rightarrow-2 v_{1}(u+v)^{-1 / 2} ; \\
(\mathrm{spl}) \rightarrow(\mathrm{w}) & u \rightarrow u_{1} / u, & v \rightarrow u v .
\end{array}
$$

The following universal substitution relates any pair equations, $(\mathrm{x})$ and $(\mathrm{X})$, denoted by the same letter, of which (x) does not contain $u, v$ explicitly:

$(\mathrm{x}) \rightarrow(\mathrm{X}) \quad u \rightarrow u_{1} \quad v \rightarrow v_{1}$.

A convenient graphical representation of the above substitutions is as follows: the systems of equations we shall denote by circles $。$ (symmetrical systems correspond to black circles •) and the substitutions of variables by arrows. As a result we get:

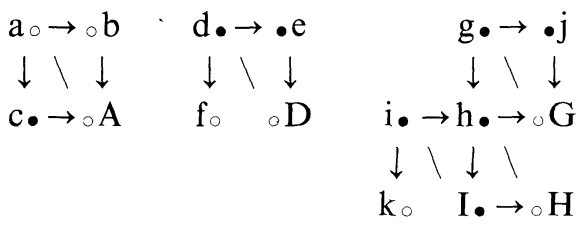

$$
\begin{aligned}
& 1 \circ \rightarrow \circ \mathrm{m} \quad \mathrm{n}_{\circ} \quad \mathrm{o} \circ \quad \mathrm{p} \circ \quad \mathrm{q} \circ \rightarrow \bullet \mathrm{r} \quad \mathrm{s} \bullet \rightarrow \circ \mathrm{t} \\
& \mathrm{u} 1 \bullet-\mathrm{U} 1 \mathrm{u} 2 \cdot \mathrm{u} 3 \text { 。 u4。 u5。 u6。 } \\
& \mathrm{V} \circ \rightarrow \circ \operatorname{lin} \quad \mathrm{spl} \circ \rightarrow \circ \mathrm{W}
\end{aligned}
$$

We notice in conclusion that it is just enough to study one of the systems from each connected graph: for the other equations of this graph all results can be reproduced via the above-given substitutions.

3. The Miura type relationships, contrary to the afore-described transformations are not invertible on a finite subset of the dynamical variables. None-the-less, they could be useful for some applications, and allow one to relate different connected graphs. A full list of all admissible Miura relations goes beyond the scope of the present paper. We confine ourselves to citing some of the relationships of this category.

As a first example we present the Miura type relations of the systems ( $\left.\mathrm{d}^{\prime}\right)$ and (i): $\left(\mathrm{d}^{\prime}\right) \Rightarrow$ (i) $\quad u \rightarrow u, \quad v_{1} \rightarrow-(u+v)^{-2} v_{1}-2(u+v)^{-1}$.

The Miura type relations of systems (e), (j) with the nonlinear Schrödinger equation we present as a composition of the following symmetrical transformation: 


$$
u_{1} \rightarrow-2(u+v)^{-1} u_{1}, \quad u+v \rightarrow \log \left[\varepsilon+2(u+v)^{-2}\right]
$$

( $\varepsilon=4,0$ in the cases (e), (j) respectively), that maps them into the system

$$
\begin{aligned}
u_{t}= & u_{2}+\left\{\frac{1}{2}[2 \varepsilon-\exp (u+v)] u_{1}^{2}+\exp (u+v) u_{1} v_{1}\right\}[\varepsilon-\exp (u+v)]^{-1} \\
& +\exp (u+v) \\
-v_{t}= & v_{2}+\left\{\frac{1}{2}[2 \varepsilon-\exp (u+v)] v_{1}^{2}+\exp (u+v) v_{1} u_{1}\right\}[\varepsilon-\exp (u+v)]^{-1} \\
& +\exp (u+v)
\end{aligned}
$$

and then with the Miura type relation:

$$
\begin{aligned}
\left(\varepsilon^{*}\right) \Rightarrow(\mathrm{b}) \quad u & \rightarrow\left\{[2 \varepsilon-2 \exp (u+v)]^{1 / 2} u_{1}+1\right\} \exp u, \\
v & \rightarrow\left\{[2 \varepsilon-2 \exp (u+v)]^{1 / 2} v_{1}+1\right\} \exp v
\end{aligned}
$$

The Miura type relationship of the system

$$
u_{t}=u_{2}+u^{2} v_{1}-u^{3} v^{2} / 2, \quad-v_{t}=v_{2}-v^{2} u_{1}-v^{3} u^{2} / 2,
$$

that is symmetrically equivalent to the derivative nonlinear Schrödinger equation (I), with the nonlinear Schrödinger equation (b) is of the form:

$\left(\mathrm{I}^{\prime}\right) \Rightarrow$ (b) $\quad u \rightarrow u_{1} \quad v \rightarrow v_{1}-v^{2} u / 2$.

To find the Miura type relations of the systems (r) and (s) we first perform the following symmetrical transformation:

$(\mathrm{s}) \rightarrow\left(\mathrm{s}^{\prime}\right) \quad \mu^{2}(u+v) \rightarrow(u+v)^{2}, \quad \mu^{2} u_{1} \rightarrow 2(u+v) u_{1}+(u+v)^{3} / 3$,

where $\mu=2(\omega-1) / 3, \omega=\exp (2 \pi i / 3)$. As a result we obtain the system

$$
\begin{aligned}
u_{t} & =u_{2}-(u+v)^{-1}\left(u_{1}^{2}+2 u_{1} v_{1}\right) / 2+\mu(u+v)^{1 / 2} v_{1} / 3+\mu^{2}(u+v)^{2} / 18, \\
-v_{t} & =v_{2}-(u+v)^{-1}\left(v_{1}{ }^{2}+2 u_{1} v_{1}\right) / 2-\mu(u+v)^{1 / 2} u_{1} / 3+\mu^{2}(u+v)^{2} / 18
\end{aligned}
$$

that is connected with Eq. (r) by the Miura type relation:

$\left(\mathrm{s}^{\prime}\right) \Rightarrow(\mathrm{r}) \quad 6 u \rightarrow\left(1-\omega^{2}\right)(u+v)^{-1 / 2} u_{1}-(u+v) / 3-\omega u$,

$$
6 v \rightarrow\left(\omega-\omega^{2}\right)(u+v)^{-1 / 2} v_{1}-\omega^{2}(u+v) / 3-\omega v .
$$

The Miura type relation of the following systems:

$$
\begin{aligned}
3 u_{t} & =-u_{2}-2 v_{2}+D\left(-u^{2}+2 v^{2}+2 u v\right), \\
-3 v_{t} & =-v_{2}-2 u_{2}+D\left(-v^{2}+2 u^{2}+2 u v\right),
\end{aligned}
$$

and

$$
u_{t}=-u_{2}+2 v_{2}, \quad v_{t}=v_{2}-2 u_{2} / 3-u^{2} / 3,
$$

which are point equivalent to Eqs. (U1) and (r) respectively, is of the form [4]:

$\left(\mathrm{U} 1^{\prime}\right) \Rightarrow\left(\mathrm{r}^{\prime}\right) \quad u \rightarrow D(2 u+v)+u v-(u+v)^{2}, \quad v \rightarrow D^{2}(u)+D(u v)-(u+v)[D(u)+u v]$.

The Miura type relation of $(u 1)$ and $(u 3)$ is of the form:

$$
\begin{aligned}
(\mathrm{u} 3) \Rightarrow(\mathrm{u} 1) \quad u_{1} & \rightarrow u_{1}-\alpha\left\{a \exp [-(u+v)]+\omega^{2} a_{1} \exp \left(-\omega u-\omega^{2} v\right)\right. \\
& \left.+\omega a_{2} \exp \left(-\omega^{2} u-\omega v\right)\right\}
\end{aligned}
$$




$$
\begin{aligned}
v_{1} & \rightarrow v_{1}-\beta\left\{a \exp [-(u+v)]+\omega a_{1} \exp \left(-\omega u-\omega^{2} v\right)\right. \\
& \left.+\omega^{2} a_{2} \exp \left(-\omega^{2} u-\omega v\right)\right\},
\end{aligned}
$$

where $2(\beta-\alpha)=1,2 \beta^{2}=\alpha$.

The Miura type relation of $(\mathrm{u} 3)$ and $(\mathrm{u} 2)$ is of the form:

$$
(\mathrm{u} 3) \Rightarrow(\mathrm{u} 2) \quad u+v \rightarrow u+u, \quad \begin{aligned}
u_{1} & \rightarrow u_{1}+\left\{a / 2 \exp [-(u+v)]-a_{1} \exp \left(-\omega u-\omega^{2} v\right)\right. \\
& \left.-a_{2} \exp \left(-\omega^{2} u-\omega v\right)\right\} / 2
\end{aligned}
$$

(the consts. $b$ and $c$ in Eq. (u2) are equal to $-3 a_{1} a_{2} / 2$ and $3 a^{2} / 32$ respectively).

\section{Commutational Representation}

A commutational representation (or the representation of the Lax type) of the nonlinear system of equations is in the basis of the inverse scattering transform. It has the form $[5,6]$ :

$$
U_{t}=D(V)+[U, V]
$$

where $U, V$ are the functions of the finite set of dynamic variables in some finite-dimensional unsolvable Lie algebra $\mathbf{A}$, it should be fulfilled as a result of the initial system and it can be considered as the matrix analogue of the local conservation laws. However, it is essential that the functions $U, V$ also contain the spectral parameter $\mu$, which is not excluded by the gauge transformations

$$
U \rightarrow g^{-1} D(g)+g^{-1} U g, \quad V \rightarrow g^{-1} g_{t}+g^{-1} V g .
$$

For a given system of equations the commutational representation (3.1) can be constructed directly from the definition (3.1) considering that $U$ and $V$ are the matrices of some fixed dimension $N \times N$. In this case a spectral parameter parametrizes a manifold of the solutions of Eq. (3.1). For each concrete equation of the matrices $U, V$ have a rather special appearance connected with the invariance of the commutational representation (3.1) relative to the action of the reduction group with elements that are the pairs for $(\mathrm{g}, g(\mu))$, where $\mathbf{g}$ is the automorphism of the Lie algebra $\mathbf{A}$, but $(g(\mu))$ is a fractional-linear transformation in the complex plane of the spectral parameter $\mu$ (see $[7,8,9]$ ). It is necessary to study the reduction group while calculating soliton solutions and formulating the corresponding Riemann problem. [7, 10]. Reduction groups are very useful in the problems of search, description and classification of the commutational representations $[7,911,12]$.

Below we shall restrict ourselves to the construction of the commutational representations for the systems of the type $(\mathrm{u})$ and the systems $(\mathrm{m}),(\mathrm{n})$. In the end we shall give references for the original papers known to us, which contain commutational representations of the other equations of the list.

1. Consider the systems of the type (u). Suppose that matrix $U$ depends on the dynamic variables $u, v$ and does not depend on $u_{1}, v_{1}$. In this case it follows directly 
from (3.1) and form $(\mathrm{u})$ that

$$
\begin{array}{ll}
V=U_{u} u_{1}-U_{v} v_{1}+W, & W=W(u, v), \\
U_{u}+U_{v v}=0, & U_{v}+U_{u u}=0, \\
W_{u}=U_{v} \theta_{v}+\left[U, U_{u}\right], & W_{v}=U_{u} \theta_{u}-\left[U, U_{v}\right], \\
{[U, W]+U_{u} z_{v}-U_{v} z_{u}=0 .} &
\end{array}
$$

It is easy to get from Eqs. (3.4) that

$$
U=\mathbf{a}_{1} \exp \left(-\omega u-\omega^{*} v\right)+\mathbf{a}_{2} \exp \left(-\omega^{*} u-\omega v\right)+\mathbf{a}_{s} \exp (-u-v)+A_{0},
$$

where $\mathbf{a}_{i}, A_{0}$ are some constant matrices. From (3.3)-(3.5) and the fact that $\theta_{u u}+\theta_{v}=\theta_{v v}+\theta_{u}=0$ (see 2,3) it is easy to get the explicit expression for $W$ :

$$
W=\left[U_{v}, U_{u}\right]+\left(U \theta-U_{u} \theta_{v}-U_{v} \theta_{u}\right) / 2-A_{0} \theta+C_{0}, \quad\left[A_{0}, U\right]=0 .
$$

$\left(C_{0}\right.$ is a constant matrix, $\left[C_{0}, U\right]=0$.) Thus, the matrix $V$ is expressed through $U$ and $\theta$ in the explicit form (3.3), (3.8). Without any loss of generality one can put $A_{0}=C_{0}=0$.

Example I. The matrix

$$
U=\left(\begin{array}{ccc}
0 & \mu_{1} \exp \left(-\omega u-\omega^{*} v\right) & 0 \\
0 & 0 & \mu_{2} \exp \left(-\omega^{*} u-\omega v\right) \\
\mu_{3} \exp (-u-v) & 0 & 0
\end{array}\right)
$$

satisfies the system of equations (3.4)-(3.6) with $\theta=z=0$. The parameters $\mu_{i}$ are free but there is an essential dependence on one parameter only $\mu=\left(\mu_{1} \mu_{2} \mu_{3}\right)^{1 / 3}-$ this parameter occurs after the gauge transformation (3.2) with $g=\operatorname{diag}\left(\left(\mu_{1} / \mu_{3}\right)^{1 / 3}\right.$, $\left.\left(\mu_{2} / \mu_{1}\right)^{1 / 3},\left(\mu_{3} / \mu_{2}\right)^{1 / 3}\right)$. The matrix $V$ is reconstructed by Eqs. (3.3), (3.8),

$$
V=\left(\begin{array}{ccc}
0 \mu_{1}\left(-\omega u_{1}+\omega^{*} v_{1}\right) \exp \left(-\omega u-\omega^{*} v\right) & \mu_{1} \mu_{2}\left(\omega-\omega^{*}\right) \exp (u+v) \\
\mu_{3} \mu_{2}\left(\omega-\omega^{*}\right) \exp \left(\omega u+\omega^{*} v\right) & 0 & \mu_{2}\left(-\omega^{*} u_{1}+\omega v_{1}\right) \exp \left(-\omega^{*} u-\omega v\right) \\
\mu_{3}\left(v_{1}-u_{1}\right) \exp (-u-v) & \mu_{1} \mu_{3}\left(\omega-\omega^{*}\right) \exp \left(\omega^{*} u+\omega v\right) & 0
\end{array}\right)
$$

Pair (3.9), (3.10) gives a commutational representation for the system (u) with $z=\theta=0$, i.e. for $(u 1)$.

Substitute the functions $\theta, z$ into (3.8), (3.6) in the form

$$
\begin{aligned}
& \theta=2 / 3\left(\omega^{*}-\omega\right) \sum_{i=1,2,3} \alpha_{i} \exp \left(-\omega^{i} u-\omega^{* i} v\right), \\
& z=\sum_{i=1,2,3}\left\{-1 / 2 \beta_{i} \exp \left(-2 \omega^{i} u-2 \omega^{* i} v\right)+\gamma_{i} \exp \left(\omega^{i} u+\omega^{* i}\right)\right\},
\end{aligned}
$$

that will allow us to embrace all the integrable cases (u1)-(u6). Collecting the coefficients at the same exponents, we get the following system of relationships for the matrices $\mathbf{a}_{i}$ :

$$
\left[\mathbf{a}_{1},\left[\mathbf{a}_{i}, \mathbf{a}_{j}\right]\right]=\alpha_{i}\left[\mathbf{a}_{i}, \mathbf{a}_{j}\right]+\beta_{i} \mathbf{a}_{j}+\gamma_{k} \mathbf{a}_{i}, \quad i \neq j \neq k \neq i .
$$

Hence, the problem of the construction of the commutational representations (3.1) for the systems of equations of the form $(u)$ is reduced tc a purely algebraic problem: it is necessary to find finite-dimensional representation of the Lie algebra, that 
generated by the elements $\mathbf{a}_{i}$ and relationships (3.12). As it has been pointed out, in order to apply the inverse scattering transform, it is necessary that this Lie algebra should be unsolvable and depend essentially on the free parameter $\mu$. The classificational result for the equations of the form $(u)$ given in II means that this problem can be only solved while fulfilling some relationships for the constants $\alpha_{i}, \beta_{i}, \gamma_{i}$. In the example 1 for the matrices $\mathbf{a}_{i}$ have the form

$$
\mathbf{a}_{1}(\mu)=\mu \mathbf{e}_{12}, \quad \mathbf{a}_{1}(\mu)=\mu \mathbf{e}_{23}, \quad \mathbf{a}_{1}(\mu)=\mu \mathbf{e}_{31},
$$

( $\mathbf{e}_{i j}$ is a matrix which has one unit in the $(i, j)$ place and the other elements vanish) and are the roots of the positive Kac-Moody subalgebra $A_{2}{ }^{(1)}$ [13].

As an example we consider this algebraic problem for the system of equations (45). In this case $\alpha_{i}=\beta_{i}=0, \gamma_{1}=\gamma_{2}=1, \gamma_{3}=\alpha$, and system (3.12) has the form

$$
\begin{array}{ll}
{\left[\mathbf{a}_{1},\left[\mathbf{a}_{1}, \mathbf{a}_{2}\right]\right]=\alpha \mathbf{a}_{1},} & {\left[\mathbf{a}_{2},\left[\mathbf{a}_{2}, \mathbf{a}_{1}\right]\right]=\alpha \mathbf{a}_{2},} \\
{\left[\mathbf{a}_{1},\left[\mathbf{a}_{1}, \mathbf{a}_{3}\right]=\mathbf{a}_{1},\right.} & {\left[\mathbf{a}_{3},\left[\mathbf{a}_{3}, \mathbf{a}_{1}\right]=\mathbf{a}_{3},\right.} \\
{\left[\mathbf{a}_{2},\left[\mathbf{a}_{2}, \mathbf{a}_{3}\right]=\mathbf{a}_{2},\right.} & {\left[\mathbf{a}_{3},\left[\mathbf{a}_{3}, \mathbf{a}_{2}\right]\right]=\mathbf{a}_{3} .}
\end{array}
$$

It is evident from these equations that matrices $\mathbf{a}_{i}$ have zero eigenvalues only. It is not difficult to check that in the matrices $2 \times 2$ Eqs. (3.14)-(3.16) do not have solutions depending essentially on a free parameter. We construct a solution in the matrices $3 \times 3$ assuming that they have a rank equal to one, and consequently they are represented by the bivectors

$$
\mathbf{a}_{1}=|a\rangle\left\langle d\left|, \quad \mathbf{a}_{2}=\right| b\right\rangle\left\langle e\left|, \quad \mathbf{a}_{3}=\right| c\right\rangle\langle f|,
$$

where |\rangle and \langle| denote a vector-column and a vector-row, respectively. Nilpotency of these matrices means that

$$
\langle d \mid a\rangle=\langle e \mid b\rangle=\langle f \mid c\rangle=0,
$$

Substituting (3.17) into (3.14)-(3.16) and taking into account (3.18) we get

$$
-2\langle d \mid b\rangle\langle e \mid a\rangle=\alpha, \quad-2\langle d \mid c\rangle\langle f \mid a\rangle=1, \quad-2\langle e \mid c\rangle\langle f \mid b\rangle=1 .
$$

We fix gauge freedom (3.2) by the choice of the vector basis

$$
|a\rangle=(1,0,0)^{\mathrm{tr}}, \quad|b\rangle=(0,1,0)^{\mathrm{tr},} \quad|c\rangle=(0,0,1)^{\mathrm{tr}},
$$

and (see (3.18))

$$
\langle d|=(0, \mu, a), \quad\langle e|=(b, 0, \mu), \quad\langle f|=(\mu, c, 0),
$$

where $\mu$ is an arbitrary parameter, the components $a, b, c$ are determined from Eqs. (3.19). As a result we obtain

$$
\mathbf{a}_{1}=\mu \mathbf{e}_{12}-\mathbf{e}_{13} /(2 \mu), \quad \mathbf{a}_{2}=\mu \mathbf{e}_{23}-\alpha \mathbf{e}_{21} /(2 \mu), \quad \mathbf{a}_{3}=\mu \mathbf{e}_{31}-\mathbf{e}_{32} /(2 \mu) .
$$

Let us point out that the dependence on the parameter $\mu$ in (3.20) is essential and these matrices cannot be reduced to a triangular form simultaneously. Thus, the matrices $\mathbf{a}_{i}$ (3.20) generate a commutational representation for the system (5).

It is easy to check that the matrices

$$
\mathbf{a}_{1}=\mu \mathbf{e}_{12}-\mathbf{e}_{32} /(4 \mu), \quad \mathbf{a}_{1}=\mu \mathbf{e}_{23}-\alpha \mathbf{e}_{21} /(4 \mu), \quad \mathbf{a}_{3}=\mu \mathbf{e}_{31}-2 \beta \mathbf{e}_{13} / \mu
$$


satisfy the system of Eqs. (3.12) with $\alpha_{i}=0, \beta_{1}=\beta_{2}=\gamma_{1}=\gamma_{2}=0, \beta_{3}=\alpha, \gamma_{3}=-2 \beta$ and give a commutational representation for the system (u2). The matrices

$$
\mathbf{a}_{1}=\mu \mathbf{e}_{12}-2 \mathbf{e}_{21} / \mu, \quad \mathbf{a}_{2}=\mu \mathbf{e}_{23}-2 \alpha \mathbf{e}_{32} / \mu, \quad \mathbf{a}_{3}=\mu \mathbf{e}_{31}-2 \mathbf{e}_{13} / \mu
$$

satisfy the system of equations (3.12) with $\alpha_{i}=\gamma_{i}=0, \beta_{1}=\alpha, \beta_{2}=\beta_{3}=1$, and give commutational representation for the system (u4).

In all cases considered the matrices $U, V, a_{i}$ have a rather special structure. This structure reflects automorphic property of the enumerated matrices relative to the action of the corresponding reduction group $G_{r}$. In this point we restrict ourselves to the consideration of the finite reduction groups, i.e. the groups generated by the transformations of the form:

$$
\begin{aligned}
& g_{1}[X(\mu)]=\mathbf{G}_{1} X\left(g_{i}(\mu)\right) \mathbf{G}_{1}{ }^{-1}, \\
& g_{2}[X(\mu)]=-\mathbf{G}_{2} X^{\operatorname{tr}}\left(g_{2}(\mu)\right) \mathbf{G}_{2}{ }^{-1},
\end{aligned}
$$

where $X(\mu)$ is the $N \times N$ matrix with the coefficient depending on $\mu ; \mathbf{G}_{1}, \mathbf{G}_{2} \in S L(N, C)$; $g_{1}(\mu), g_{2}(\mu)$ are fractional linear transformations of the complex plane $\mu$ (for a more detailed and gauge invariant definition of the reduction group see [7]). We shall call the matrix $X(\mu)$ an automorphic one relative to the reduction group $G_{r}$, if

$$
g[X(\mu)]=X(\mu)
$$

for all $g \in G_{r}$. It is convenient to construct automorphic matrices via the averaging over the reduction group

$$
\langle X(\mu)\rangle=\Sigma g[X(\mu)] .
$$

For instance, the matrices $U(3.9), V(3.10)$ are automorphic relative to the group $G_{r} \simeq \mathbf{Z}_{3}$, generated by the transformation

$$
q[X(\mu)]=Q X(q(\mu)) Q^{-1}, \quad Q=\operatorname{diag}\left(\omega, \omega^{*}, 1\right), \quad q(\mu)=\omega \mu, \quad \omega=\exp (2 \pi \mathrm{i} / 3) .
$$

They are obtained by averaging over this reduction group of the following matrices:

$$
U=\mu A, \quad V=\mu^{2} B+\mu C+F,
$$

( $A, B, C, F$ are the matrices of a general form).

We turn to the consideration of the commutational representations invariant relative to the finite reduction groups which are a stationary subgroup of the point $\mu=\infty$ generated by transformation (3.24). In this case we can restrict ourselves to the averaging of the matrices $U$ of the form (3.29) $\left(\mu_{1}=\mu_{2}=\mu_{3}=\mu\right)$. Here the corresponding matrices $\mathbf{a}_{i}$ are obtained by the averaging of the matrices $\mathbf{a}_{i}(\mu)(3.18)$. The finite groups of the fractional-linear transformations of the complex plane are exhausted by the group rotations $\left(\mathbf{Z}_{N}\right)$, dihedrons $\left(\mathbf{D}_{N}\right)$ and regular polytopes. We have already considered the rotation group $\mathbf{Z}_{3}$-it results in the commutational representation (3.9) (3.10) for the system (w2).

The dihedron group $\mathbf{D}_{3}$ has two nonequivalent realization (representations as the reduction group):

i) The generators $q, k\left(q^{3}=k^{2}=(q k)^{2}=i d\right)$ are represented by the transformations 


$$
\begin{array}{ll}
q[X(\mu)]=Q X(q(\mu)) Q^{-1}, & Q=\operatorname{diag}\left(\omega, \omega^{*}, 1\right), \quad q(\mu)=\omega \mu, \\
\left.k[X(\mu)]=-X^{\operatorname{tr}}(-\delta / \mu)\right), & \delta \in C .
\end{array}
$$

The averaging of the matrices $\mathbf{a}_{i}(\mu) / 3$ (3.13) over the reduction group with the generators (3.26) (3.27) yields (cf. (3.22))

$$
\mathbf{a}_{1}=\mu \mathbf{e}_{12}+\delta \mathbf{e}_{21} / \mu, \quad \mathbf{a}_{2}=\mu \mathbf{e}_{23}+\delta \mathbf{e}_{32} / \mu, \quad \mathbf{a}_{3}=\mu \mathbf{e}_{31}+\delta \mathbf{e}_{13} / \mu .
$$

The matrices $\mathbf{a}_{i}$ (3.28) satisfy relationships (3.12) with $\alpha_{i}=\gamma_{i}=0, \beta_{i}=\delta$ and, thus, give commutational representations for the system of the type $(\mathrm{u} 4)$.

ii) Let us represent the generators $q, k$ by transformations (3.26) and

$$
h[X(\mu)]=H X(-2 \delta / \mu) H, \quad H=\left(\begin{array}{ccc}
0 & 0 & \tau \\
0 & 1 & 0 \\
1 / \tau & 0 & 0
\end{array}\right), \delta, \tau \in C .
$$

The averaging of matrices $\mathbf{a}_{i}(\mu) / 3$ (3.13) over the reduction group with generators (3.26) (3.29) yields (cf. (3.21))

$$
\mathbf{a}_{1}=\mu \mathbf{e}_{12}-\delta \mathbf{e}_{32} /(\tau \mu), \quad \mathbf{a}_{2}=\mu \mathbf{e}_{23}-\delta \mathbf{e}_{21} /(\tau \mu), \quad \mathbf{a}_{3}=\mu \mathbf{e}_{31}-\delta \mathbf{e}_{13} / \mu .
$$

The matrices $\mathbf{a}_{i}(\mu)$ (3.30) satisfy the commutational relationships (3.12) with $\alpha_{i}=0$, $\beta_{1}=\beta_{2}=\gamma_{1}=\gamma_{2}=0, \beta_{3}=-\delta, \gamma_{3}=4 \delta / \tau$. They generate a commutational representation for the system (u2) with $\alpha=4 \delta \tau, \beta=\delta / 2$.

As generators of the tetrahedron group one can choose $q, k\left(q^{3}=k^{2}=(q k)^{3}=i d\right)$. Let $q$ have representation (3.26) and

$$
t[X(\mu)]=T X(t(\mu)) T, \quad T=1 / 3\left(\begin{array}{rrr}
-1 & 2 & 2 \\
2 & -1 & 2 \\
2 & 2 & -1
\end{array}\right), t(\mu)=(\mu+2) /(\mu-1) .
$$

The averaging of the matrices $\mathbf{a}_{i}(\mu) / 3$ (3.13) over the group generated by transformations (3.26) (3.31) yields

$$
\begin{gathered}
\mathbf{a}_{T 1}(\mu)=\left(\begin{array}{rrr}
-2 a & \mu+b & -2 c \\
4 c & -2 a & 4 b \\
4 b & -2 c & 4 a
\end{array}\right), \quad \mathbf{a}_{T 2}(\mu)=\left(\begin{array}{rrr}
4 a & 4 b & -2 c \\
-2 c & -2 a & \mu+b \\
4 b & 4 c & -2 a
\end{array}\right), \\
\mathbf{a}_{T 3}(\mu)=\left(\begin{array}{rrr}
-2 a & 4 b & 4 c \\
-2 c & 4 a & 4 b \\
\mu+b & -2 c & -2 a
\end{array}\right),
\end{gathered}
$$

where

$$
\begin{aligned}
& a=\left(t(\mu)+t\left(\omega^{*} \mu\right)+t(\omega \mu)\right) / 9=\left(\mu^{3}+2\right) /\left(3 \mu^{3}-3\right), \\
& b=\left(t(\mu)+\omega t\left(\omega^{*} \mu\right)+\omega^{*} t(\omega \mu)\right) / 9=\mu /\left(\mu^{3}-1\right), \\
& c=\left(t(\mu)+\omega^{*} t\left(\omega^{*} \mu\right)+\omega t(\omega \mu)\right) / g=\mu^{2} / 9\left(\mu^{3}-1\right) .
\end{aligned}
$$

One can check that the matrices $\mathbf{a}_{T i}(\mu)$ (3.32) satisfy commutational relationships (3.12) with $\alpha_{i}=4, \gamma_{i}=\beta_{i}=0$ and hence, give a commutational representation for the system of the type (u3). 
Octahedron group can be represented by the transformations $q, t(3.26),(3.31)$ and

$$
\left.k[X(\mu)]=-X^{\operatorname{tr}}(-2 / \mu)\right) .
$$

It is evident that the matrices $\mathbf{a}_{i}$ corresponding to this group are obtained from the matrices $\mathbf{a}_{T i}(\mu)(3.22)$ as follows:

$$
\mathbf{a}_{i}=\mathbf{a}_{T i}(\mu)-\mathbf{a}_{T i}{ }^{\mathrm{tr}}(-2 / \mu) .
$$

One can prove that matrices $\mathbf{a}_{i}$ (3.34) satisfy commutational relationships (3.12) with $\alpha_{i}=12, \gamma_{i}=\beta_{i}=32$, and hence, generate a commutational representation for the system of the type (u6).

2. Consider systems (m), (n). System (m) represents a well-known Landau-Lifshitz model with a biaxial anisotropy. The commutational representation for it has been practically simultaneously obtained in papers $[14,15]$. The author of the first paper proceeded directly from the definition (3.1), in the second one this system of equations and its commutational representation have been obtained as a result of the quasi-classical transition from the exactly solvable quantum XYZ model. Here following paper [10] (for detail see [16]) we get this commutational representation having averaged the matrices

$$
\begin{aligned}
& U=i / \mu \Sigma \sigma_{k} S_{k}, \\
& V=i \Sigma \sigma_{k}\left(A_{k} / \mu+2 S_{k} / \mu^{2}\right)
\end{aligned}
$$

(here $A_{k}=\Sigma \varepsilon_{k l m} S_{1} S_{m x}$ ), corresponding to the Heisenberg model over the infinite reduction group $G_{r}$, given by the generators

$$
\begin{aligned}
& g_{1}[X(\mu)]=\sigma_{3} X(\mu+\omega) \sigma_{3}, \quad \operatorname{Im}\left(\omega / \omega^{\prime}\right) \neq 0, \\
& g_{2}[X(\mu)]=\sigma_{1} X\left(\mu+\omega^{\prime}\right) \sigma_{1} .
\end{aligned}
$$

As a result we obtain

$$
\begin{aligned}
& U=i \Sigma \sigma_{k} W_{k}^{1}(\mu) S_{k}, \\
& V=i \Sigma \sigma_{k} W_{k}^{1}(\mu) S_{1} S_{m x} \varepsilon_{k l m}+2 i \Sigma \sigma_{k} W_{k}^{2}(\mu) S_{k},
\end{aligned}
$$

where $W_{k}^{a}(\mu)$ are elliptical functions which expressions through the Weierstrass function have the form

$$
\begin{aligned}
W_{1}^{1}(\mu) & =\zeta(\mu)+\zeta\left(\mu+\omega^{\prime}\right)-\zeta(\mu+\omega)-\zeta\left(\mu+\omega+\omega^{\prime}\right)+2 \zeta(\omega), \\
W^{1}{ }_{2}(\mu) & =\zeta(\mu)-\zeta\left(\mu+\omega^{\prime}\right)-\zeta(\mu-\omega)+\zeta\left(\mu+\omega+\omega^{\prime}\right), \\
W^{1}{ }_{3}(\mu) & =\zeta(\mu)-\zeta\left(\mu+\omega^{\prime}\right)+\zeta(\mu+\omega)-\zeta\left(\mu+\omega+\omega^{\prime}\right)+2 \zeta\left(\omega^{\prime}\right), \\
W^{a+1}{ }_{k}(\mu) & =(-1)^{a} / a ! d^{a}\left(W^{1}{ }_{k}(\mu)\right) / d \mu^{a},
\end{aligned}
$$

and $\left(W^{1}{ }_{k}(\mu)\right)^{2}-\left(W^{1}{ }_{m}(\mu)\right)^{2}=\left(J_{m}-J_{k}\right) / 2, J_{k} \in C$. It is easy to check that (3.1), (3.38) yield a system of equations

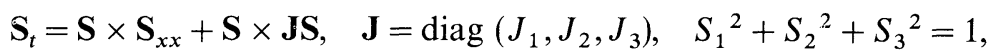

which is reduced to the form (m) by the substitution (1.14). 
If we substitute $\mu^{2}$ instead of $\mu$ in matrices (3.35), (3.36), then their averaging over the reduction group $G_{r}$ (3.37) gives a commutational representation for the system

$$
\mathbf{S}_{t}=\mathbf{S} \times \mathbf{S}_{x x}-(\mathbf{S}(\mathbf{S}, \mathbf{J S}))_{x} / 4+\mathbf{J S}_{x} / 2
$$

related by the substitution (1.14) to system (n) in which

$$
\begin{aligned}
& U=i \Sigma \sigma_{k} W_{k}^{2}(\mu) S_{k}, \\
& V=i \Sigma \sigma_{k} W_{k}^{2}(\mu)\left[-J_{k} S_{k} / 2+\Sigma S_{1} S_{m x} \varepsilon_{k l m}\right]-2 i \Sigma \sigma_{k} W_{k}^{4}(\mu) S_{k} .
\end{aligned}
$$

Representation (3.1), (3.42) has been obtained in paper [17] by another methodthe author proceeded from the infinite-dimensional Lie algebra of a strictly unit growth as first occurred in reports $[11,12]$. We point out that the elliptical functions $W^{2 a}{ }_{k}(\mu)$ in (3.42) can be substituted for the rational functions of a new spectral parameter. Really, from definition (3.39) one can get easily the following relationships between the functions $X_{k}=W^{2}{ }_{k}(\mu), Y_{k}=W^{4}{ }_{k}(\mu)$ :

$$
\begin{aligned}
& X_{i} X_{j}=Y_{k}-J_{k} X_{k} / 4, \quad k \neq i \neq j \neq k, \\
& X_{i} Y_{j}-X_{j} Y_{i}=\left(J_{i}-J_{j}\right) Y_{k} / 4+\left(J_{i}{ }^{2}-J_{j}{ }^{2}\right) X_{k} .
\end{aligned}
$$

The intersection of quadrics (3.43) yields a curve of the zero genus which can be simply uniformized by rational functions. Here a uniformizing variables will serve as a new spectral parameter. But from the practical viewpoint the representation obtained by averaging over the reduction group is the most convenient one since it allows to integrate a nonlinear system by a well-known scheme (see for instance [16]).

3. In conclusion we give a list of the original papers where one can find commutational representations of the known systems for the equations of our list: (A)-[18]; (b)-[19]; (c)-[20]; (d')-[21]; (e)-[22]; (H)-[23]; (h') $\Leftrightarrow\left(\mathrm{h}^{\prime \prime}\right)-[24] ;(\mathrm{I})-[25]$; (Q)-[13]; (r)-[26]; (u1) - [26]; (U1), (u4)-[7].

\section{References}

1. Mikhailov, A. V., Shabat, A. B.: Integrability conditions for systems of two equations of the form $\mathbf{u}_{t}=A(\mathbf{u}) \mathbf{u}_{x x}+\mathbf{F}\left(\mathbf{u}, \mathbf{u}_{x}\right)$. I. Teor. Mat. Fiz. 62, 163-185 (1985)

2. Mikhailov, A. V., Shabat, A. B.: Integrability conditions for systems of two equations of the form $\mathbf{u}_{t}=A(\mathbf{u}) \mathbf{u}_{x x}+\mathbf{F}\left(\mathbf{u}, \mathbf{u}_{x}\right)$. II. Teor. Mat. Fiz. 66, 47-65 (1986)

3. Shabat, A. B., Yamilov, R. I.: Preprint Ufa (1985)

4. Sokolov, V. V., Shabat, A. B.: Funct. Anal. Appl. 14, 79-80 (1980)

5. Zakharov, V. E., Shabat, A. B.: Funct. Anal. Appl. 13(3), 13-22 (1979)

6. Zakharov, V. E. Mikhailov, A. V.: ZhETF, 74(6), 1953-1973 (1978)

7. Mikhailov, A. V.: In: Soliton theory, proceeding of the Soviet-American symposium on soliton theory. Kiev, USSR, 1979, Manakov, S. V., Zakharov, V. E. (eds.): The reduction problem and the inverse scattering method. Physica 3D 1 \& 2, 73-117 (1981)

8. Mikhailov, A. V.: Pisma v ZhETF 433-448 (1979)

9. Mikhailov, A. V.: Pisma v ZhETF 32, 2, 187-192 (1980)

10. Mikhailov, A. V.: The Landau-Lifschitz equation and the Riemann boundary problem on a tours. Phys. Lett. 92A 2, 51-55 (1982)

11. Mikhailov, A. V.: Algebraic and analytic structure of $\mathrm{L}-\mathrm{A}$ pairs. Basic classifications. Report on International Workshop. Kiev 1983 
12. Mikhailov, A. V.: The reduction group average and filtered Lie algebras of finite growth. Report on the NORDITA-USSR. Workshop, Copenhagen 1982

13. Drinfeld, V. G., Sokolov, V. V.: Itogi Nauki-Tekh. Ser. Sovrem. Probl. Mat. 24, Moskva, VINITI, 81-180 (1984)

14. Borovik, A. Ye.: Doktorskaya dissertaciya. Kharkov 1981.

15. Sklyanin, E. K.: Sovremenniye problem teorii magnetisma. Kiev, Naukova Dumka str. 12 (1986)

16. Mikhailov, A. V.: In: Solitons, modern problems in condensed matter sciences, Vol 17. Trullinger S. F., Zakharov, V. E., Pokrovskii, V. L. (eds.). Amsterdam: North Holland, pp. 623-690 (1986)

17. Bar'yakhtar, V. G., Belokolos, E. D., Golod, P. I. (see [15] p. 30).

18. Reyman, A. G.: Differenz. geometriya, gruppi Lie i mechanika. Zap. Nauchn. Semin. LOMI, 95, L. Nauka, 3-54 (1980)

19. Zakharov, V. E., Shabat, A. B.: Exact theory of two-dimensional self-focusing and one-dimensional self-modulation of waves in nonlinear media. Sov. Phys. JETP 34, 62 (1972)

20. Kaup, D. J.: Finding eigenvalue problems for solving nonlinear evolution equations. Progr. Theor. Phys., 54(1), 72-78 (1975)

21. Kundu, A.: Landau-Lifshitz and higher-order nonlinear systems gauge generated from nonlinear Schrödinger type equations. J. Math. Phys. 25, 3433 (1984)

22. Borovik, A. E.: Pisma v ZhETF 28(10), 629-632 (1978)

23. Levi, D.: Nonlinear differential difference equations as Bäcklund transformations. J. Phys. A: Math. Gen. 14, 1083-1098 (1981)

24. Takhtajn, L. A.: Integration of the continuous Heisenberg spin chain through the inverse scattering method. Phys. Lett. 64A 2, 235-237 (1977)

25. Kaup, D. J., Newell, A. C.: An exact solution for a derivative nonlinear Schrödinger equation, J. Math. Phys. 19, 798-801 (1978)

26. Zakharov, V. E.: ZhETF 65(1), 219-225 (1973)

Communicated by G. Parisi

Received March 15, 1987 
